Case Report

\title{
A Case of Spontaneous Tumor Lysis Syndrome in a Patient with Ovarian Cancer
}

\author{
Kazuhiro Okamoto, ${ }^{1}$ Toshifumi Kinoshita, ${ }^{1}$ Miyuki Shimizu, ${ }^{1}$ Isoji Okura, ${ }^{1}$ \\ Akinori Kawada, ${ }^{1}$ Koichi Mizobuchi, ${ }^{2}$ and Midori Ando ${ }^{2}$ \\ ${ }^{1}$ Department of Obstetrics and Gynecology, Kagawa Rosai Hospital, 3-3-1 Zyotocho, Marugame-shi, Kagawa-ken 763-8502, Japan \\ ${ }^{2}$ Department of Pathology, Kagawa Rosai Hospital, 3-3-1 Zyotocho, Marugame-shi, Kagawa-ken 763-8502, Japan
}

Correspondence should be addressed to Kazuhiro Okamoto; kazuhiro.errore@gmail.com

Received 3 April 2015; Accepted 10 June 2015

Academic Editor: Giampiero Capobianco

Copyright (C) 2015 Kazuhiro Okamoto et al. This is an open access article distributed under the Creative Commons Attribution License, which permits unrestricted use, distribution, and reproduction in any medium, provided the original work is properly cited.

\begin{abstract}
Tumor lysis syndrome (TLS) is a potentially life-threating complication of tumors or chemotherapy treatment. TLS commonly occurs in hematological malignancies, but it is very rare in patients with a solid tumor. In cases of solid tumors, TLS usually occurs spontaneously and after the initiation of anticancer therapy, and it has a high mortality rate. We present the novel case of a $62-$ year-old woman with an ovarian tumor who spontaneously developed TLS. Surgical reduction of the tumor mass vastly improved her condition. She showed no sign of tumor recurrence 8 months after treatment. As TLS is life-threatening, successful treatments must be seriously considered.
\end{abstract}

\section{Introduction}

Tumor lysis syndrome (TLS) is characterized by the massive lysis of malignant cells and the rapid release of intracellular components into the systemic circulatory system. It requires immediate intervention and is sometimes lethal [1]. It is typically associated with hematopoietic malignancies, but it is rare in patients with solid tumors [2]. In cases of solid tumors, TLS usually occurs spontaneously or after antineoplastic treatment [3]. It has a mortality rate as high as $40 \%$ due to inadequate preventive treatment and delayed curative treatment [4]. There are few previous reports on TLS in patients with ovarian cancer, and all were associated with chemotherapy [5-10]. Herein, we are the first to report on a case of ovarian cancer with spontaneous TLS.

\section{Case Presentation}

The patient was a 62-year-old nonparous woman with no history of a gynecological disease. She had an enlarged abdomen during the 2 months preceding her visit to our hospital, and she had lower abdominal pain 2 days before her visit. She was transported to our hospital by ambulance and complained of disabling back pain. An abdominal examination revealed a palpable mass in the right lateral region of her abdomen. Transabdominal echography and computed tomography revealed a multilocular-solid tumor ( $>20 \mathrm{~cm}$ in diameter) in her pelvis, and massive ascites extended into the liver and spleen (Figure 1). Blood analysis showed hyperuricemia, hyperkalemia, hyperphosphatemia, and increased levels of plasma creatinine (Table 1). These findings fulfilled the diagnostic criteria of TLS.

Following admission, the patient had anuria that responded poorly to aggressive fluid administration. We thought that her presentation could not be explained only by fluid shifts. Two days after admission, laboratory data indicated that her condition had worsened. Her respiratory status declined, and she was placed on a respirator. She also received a vasopressor to maintain her blood pressure. We decided to surgically reduce the amount of the tumor mass and to drain her abdomen in order to improve her condition. Abdominal bilateral salpingo-oophorectomy, hysterectomy, and abdominal drainage were performed (Figure 2).

We made a midline skin excision extending from the symphysis pubis to the right side of the umbilicus. The abdominal cavity was adequately exteriorized, and 3,300 mL 


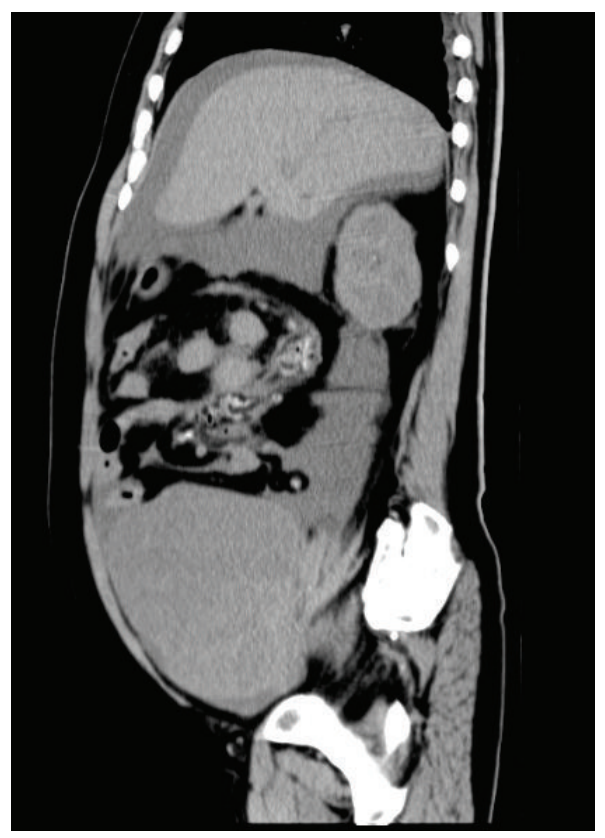

(a)

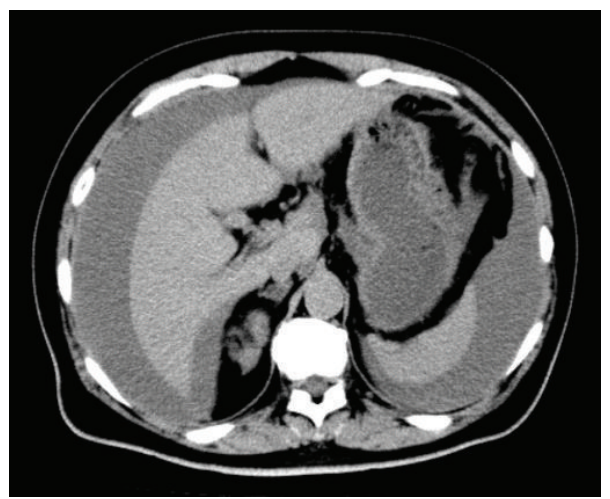

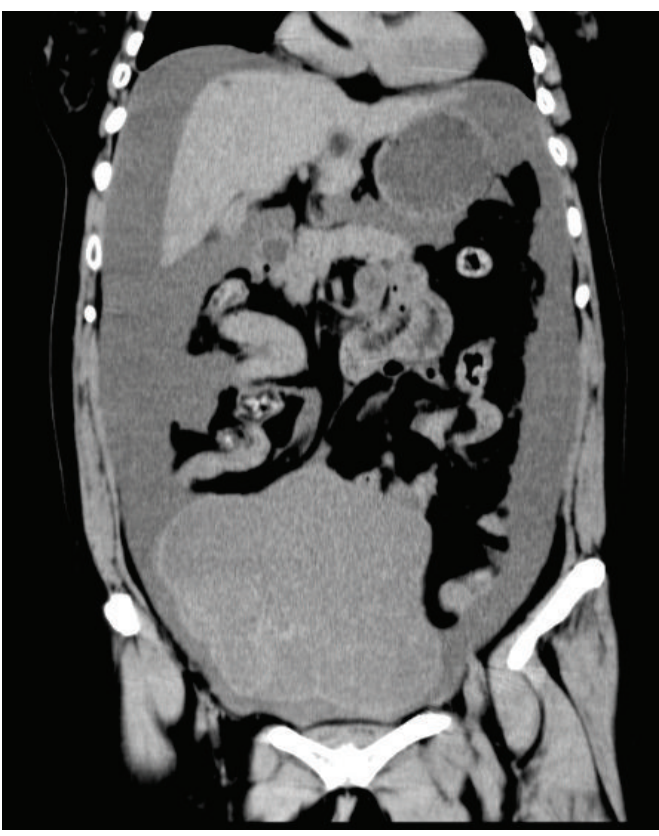

(b)

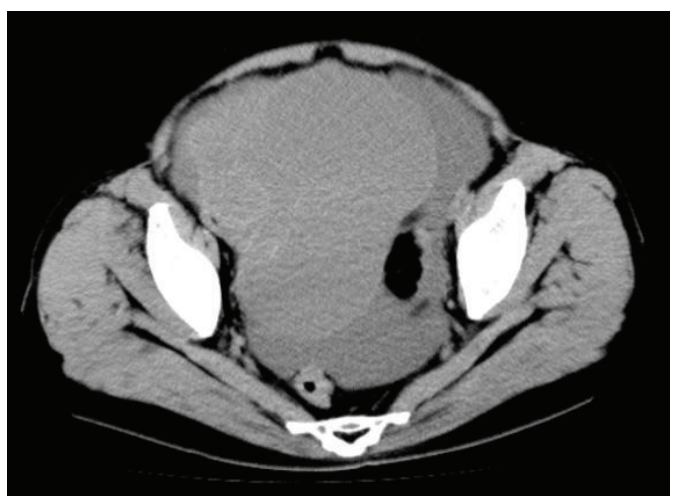

(c)

FIGURE 1: Abdominal plane computed tomography reveals a large pelvic mass with massive ascites that reach the liver and spleen. (a) Sagittal section; (b) frontal section; (c) coronal section.

of bloody, viscous fluid was suctioned. The large mass in the right ovary was extracted with the uterus and left ovary, which were macroscopically normal. The abdominal cavity was washed with saline to remove tumor tissue that adhered to the thickened, hyperemic peritonea. A drainage tube was placed in the upper abdomen, and the abdomen was closed. The patient received intensive care postoperatively, and her condition improved. The expression levels of biochemical markers returned to normal.

Pathologic examination of the neoplasm from the right ovary showed a well-differentiated (grade 1) endometrioid adenocarcinoma with extensive necrosis and capsular rupture (Figure 3). Cytology of the ascitic fluid showed no malignant cells. There were no remarkable changes in the macroscopic appearance of the left ovary or uterus. The final surgical pathologic diagnosis was stage IC ovarian endometrioid adenocarcinoma.
Postoperatively, the patient received six cycles of combination chemotherapy, which consisted of docetaxel and carboplatin. No signs of recurrence were observed at the 8month follow-up.

\section{Discussion}

TLS is a potentially life-threatening complication of malignancies. It results from massive cytolysis of malignant tumor cells, either spontaneous or chemotherapy-induced, and the release of intracellular components into the circulatory system. TLS is characterized by hyperuricemia, hyperphosphatemia, hyperkalemia, hypocalcemia, increased plasma creatinine levels, and other abnormalities that can lead to acute renal dysfunction, fatal cardiac arrhythmia, central nervous system toxicity, and death [1]. The most widely accepted diagnostic criteria of TLS are provided in 


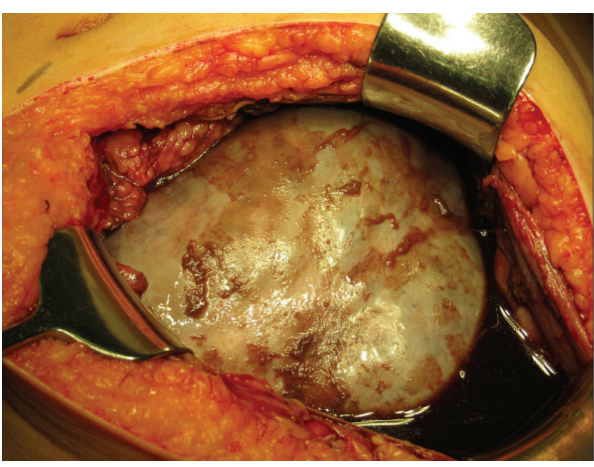

(a)

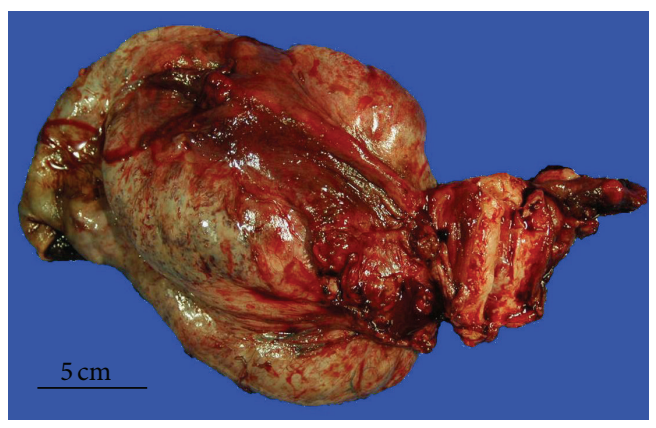

(c)

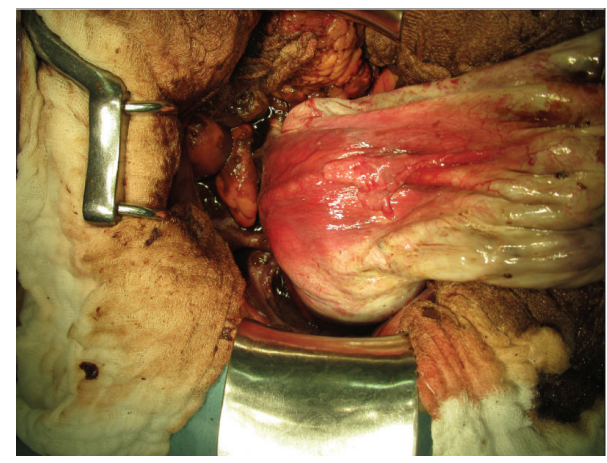

(b)

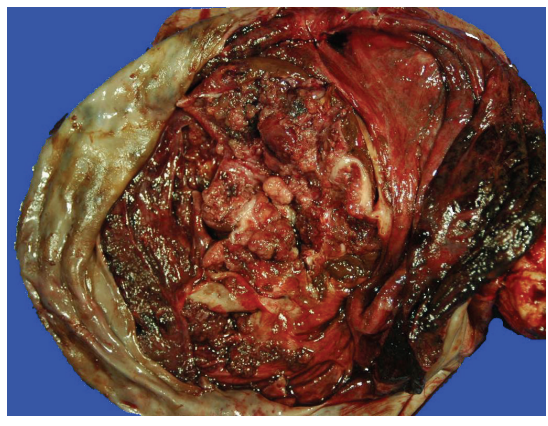

(d)

Figure 2: Operative findings. (a) The right ovary is markedly enlarged and contains a carcinoma. (b) The large ovarian tumor is removed via a vertical midline incision after abdominal drainage is performed. (c) The excised tumor mass is $20 \mathrm{~cm}$ in diameter. The uterus and left ovary are macroscopically normal. (d) The tumor mass contains septations and papillary solid lesions.

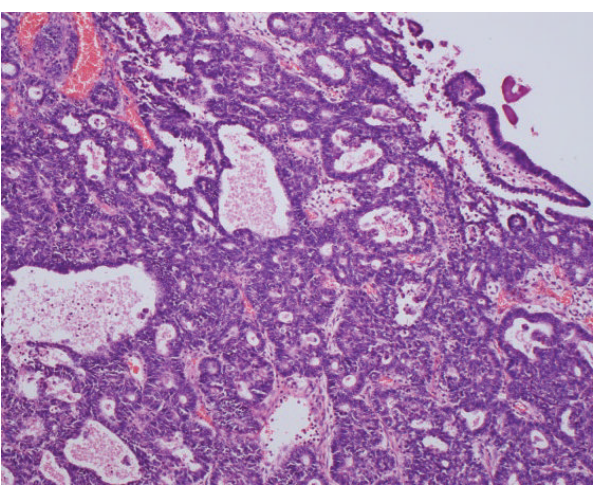

(a)

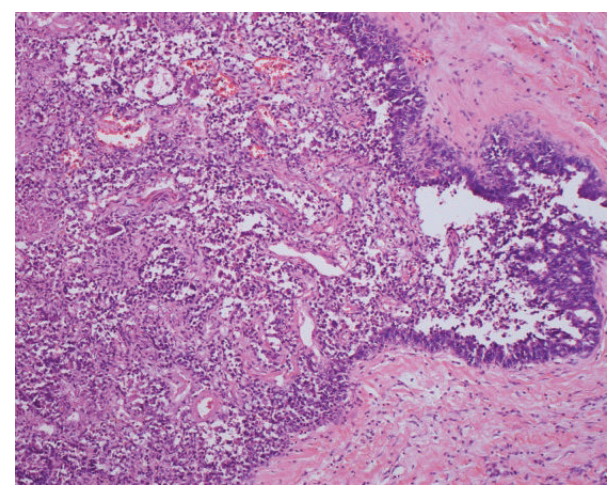

(b)

Figure 3: Pathological findings. (a) A grade 1 endometrioid adenocarcinoma is characterized by glandular patterns resembling those of the endometrium. (b) Extensive necrosis of the endometrioid carcinoma (left side). (a-b) Hematoxylin and eosin staining (magnification, $\times 100$ ).

the Cairo-Bishop definition of laboratory TLS [1]. In our case, these criteria were met. TLS typically occurs in hematological malignancies, especially in acute lymphocytic leukemia and Burkitt's lymphoma, but it rarely occurs in solid tumors [2].

Potential risk factors of TLS in cases with solid tumors include liver metastasis, high lactate dehydrogenase and uric acid levels, renal insufficiency, prior treatment with nephrotoxic drugs, infection, and dehydration [11]. Solid tumors that may spontaneously rupture typically have a rapid growth, high volume, or chemosensitivity [3]. Six cases of ovarian cancer complicated by TLS have been reported in the literature, and all were associated with chemotherapy [5-10]. Conversely, we report on a novel case of spontaneous TLS in a patient with ovarian cancer. In this case, the risk factor for TLS appeared to be the large volume of the tumor. During surgery, we found that the tumor had ruptured, and the pathologic examination revealed extensive necrosis of the tumor. The rapid growth rate of the tumor presumably accounted for its large volume. 
TABLE 1: Patient's laboratory data on admission.

\begin{tabular}{lclc}
\hline CBC & \multicolumn{3}{c}{ Blood chemistry } \\
WBC & $14,300 / \mu \mathrm{L}$ & BUN & $47 \mathrm{mg} / \mathrm{dL}$ \\
RBC & $451 \times 10^{4} / \mu \mathrm{L}$ & $\mathrm{Cr}$ & $2.98 \mathrm{mg} / \mathrm{dL}$ \\
$\mathrm{Hb}$ & $14.4 \mathrm{~g} / \mathrm{dL}$ & $\mathrm{UA}$ & $8.1 \mathrm{mg} / \mathrm{dL}$ \\
$\mathrm{Ht}$ & $41.2 \%$ & $\mathrm{Na}$ & $132 \mathrm{mEq} / \mathrm{L}$ \\
Platelet & $32.9 \times 10^{4} / \mu \mathrm{L}$ & $\mathrm{K}$ & $4.9 \mathrm{mEq} / \mathrm{L}$ \\
Serological test & & $\mathrm{Cl}$ & $91 \mathrm{mEq} / \mathrm{L}$ \\
CRP & $22.5 \mathrm{mg} / \mathrm{dL}$ & $\mathrm{P}$ & $8.7 \mathrm{mg} / \mathrm{dL}$ \\
Coagulation & & Glucose & $435 \mathrm{mg} / \mathrm{dL}$ \\
FDP & $16.3 \mu \mathrm{g} / \mathrm{mL}$ & CEA & $32.9 \mathrm{mg} / \mathrm{mL}$ \\
D-dimer & $6.9 \mu \mathrm{g} / \mathrm{mL}$ & CA19-9 & $1,999 \mathrm{U} / \mathrm{mL}$ \\
& & CA125 & $4,325.1 \mathrm{U} / \mathrm{mL}$ \\
\hline
\end{tabular}

CBC: complete blood count, WBC: white blood cell, and RBC: red blood cell. $\mathrm{Hb}$ : hemoglobin, Ht: hematocrit.

CRP: C-reactive protein, FDP: fibrin/fibrinogen degradation products, BUN: blood urea nitrogen, Cr: creatinine, UA: urinary acid, Na: sodium, K: potassium, Cl: chlorine, P: phosphorus, CEA: carcinoembryonic antigen, CA19-9: carbohydrate antigen 19-9, and CA125: carbohydrate antigen 125.

Treatment of TLS consists of aggressive hydration, the correction of electrolyte disturbances, and uric acid-lowering therapy [3]. The mortality rate of TLS in cases with solid tumors is higher than in cases with nonsolid malignancies, and it is reportedly as high as $40 \%$ [4]. Greater consideration of preventive treatments for TLS and the prompt treatment of TLS may aid in decreasing this rate. In the present case, the patient's condition worsened despite continuous intensive care. She required a respirator for breathing and a vasopressor to maintain her blood pressure. Because we thought that death was imminent, we performed surgery to reduce the amount of the tumor mass and to drain the abdomen in order to improve her condition. Postoperatively, her condition improved. Complete cytoreductive surgery removed most of the lysed tumor, which may have largely contributed to her improved condition. When nonsurgical treatment of TLS elicits a poor response or no response, debulking surgery seems to be a viable option.

TLS is a relatively rare event in patients with solid cancers. Nevertheless, clinicians should remember that such patients may develop this lethal complication in response to chemotherapy or even spontaneously.

\section{Conflict of Interests}

The authors declare that there is no conflict of interests regarding the publication of this paper.

\section{References}

[1] M. S. Cairo and M. Bishop, "Tumour lysis syndrome: new therapeutic strategies and classification," British Journal of Haematology, vol. 127, no. 1, pp. 3-11, 2004.

[2] A. Will and E. Tholouli, "The clinical management of tumour lysis syndrome in haematological malignancies," British Journal of Haematology, vol. 154, no. 1, pp. 3-13, 2011.
[3] A. E. Mirrakhimov, A. M. Ali, M. Khan, and A. Barbaryan, "Tumor lysis syndrome in solid tumors: an up to date review of the literature," Rare Tumors, vol. 6, no. 2, pp. 668-676, 2014.

[4] TLS guidance, Japanese Society of Medical Oncology, pp. 10-12, 2013, http://jsmo.or.jp/news/jsmo/doc/20130716.pdf.

[5] S. F. A. Bilgrami and B. G. Fallon, "Tumor lysis syndrome after combination chemotherapy for ovarian cancer," Medical and Pediatric Oncology, vol. 21, no. 7, pp. 521-524, 1993.

[6] J. K. Chan, S. S. Lin, D. S. McMeekin, and M. L. Berman, "Patients with malignancy requiring urgent therapy: CASE 3. Tumor lysis syndrome associated with chemotherapy in ovarian cancer," Journal of Clinical Oncology, vol. 23, no. 27, pp. 67946795, 2005.

[7] T. Yahata, N. Nishikawa, Y. Aoki, and K. Tanaka, “Tumor lysis syndrome associated with weekly paclitaxel treatment in a case with ovarian cancer," Gynecologic Oncology, vol. 103, no. 2, pp. 752-754, 2006.

[8] M. Doi, Y. Okamoto, M. Yamauchi, H. Naitou, and K. Shinozaki, "Bleomycin-induced pulmonary fibrosis after tumor lysis syndrome in a case of advanced yolk sac tumor treated with bleomycin, etoposide and cisplatin (BEP) chemotherapy," International Journal of Clinical Oncology, vol. 17, no. 5, pp. 528531, 2012.

[9] T. Cui, C. Wang, H. He, G. Shi, and L. Hu, "A rare case of ovarian Burkitt lymphoma associated tumor lysis syndrome," European Journal of Gynaecological Oncology, vol. 31, no. 2, pp. 209-210, 2010.

[10] M. Camarata, R. Davies, S. Copley, and S. Blagden, “Tumour lysis syndrome in a patient with intravascular spread from a recurrent epithelial ovarian cancer," BMJ case reports, 2013.

[11] C. Gemici, "Tumour lysis syndrome in solid tumours," Clinical Oncology, vol. 18, no. 10, pp. 773-780, 2006. 


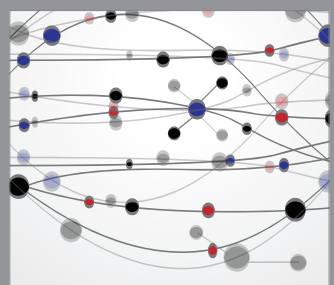

The Scientific World Journal
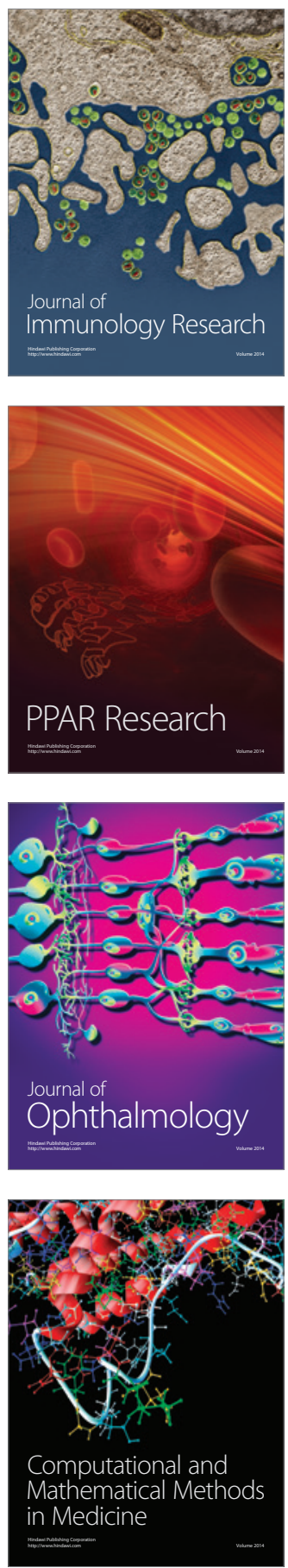

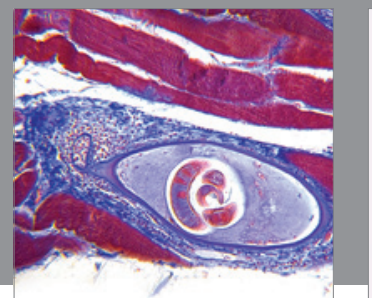

Gastroenterology

Research and Practice
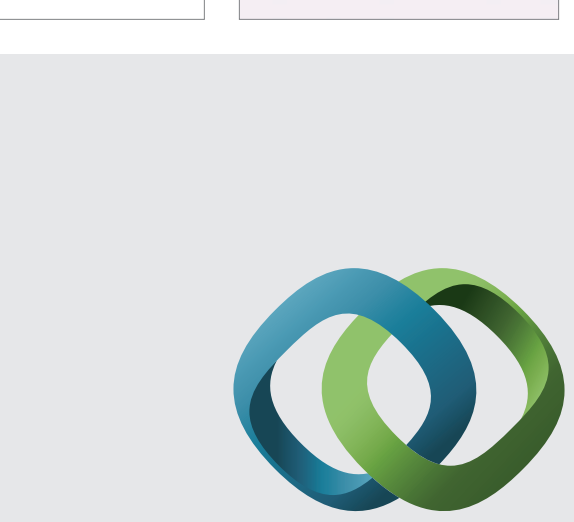

\section{Hindawi}

Submit your manuscripts at

http://www.hindawi.com
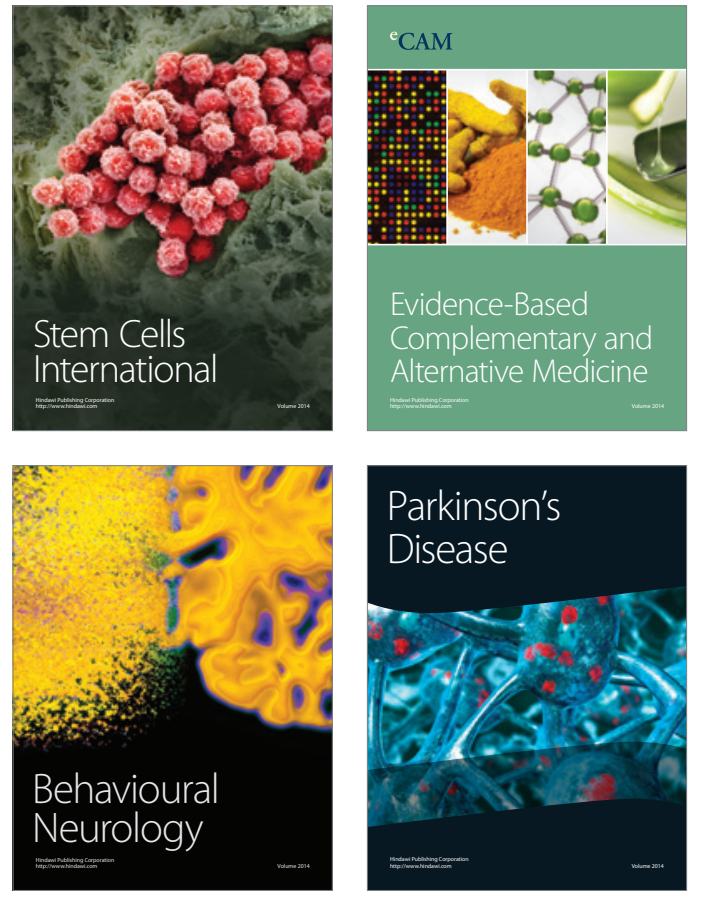
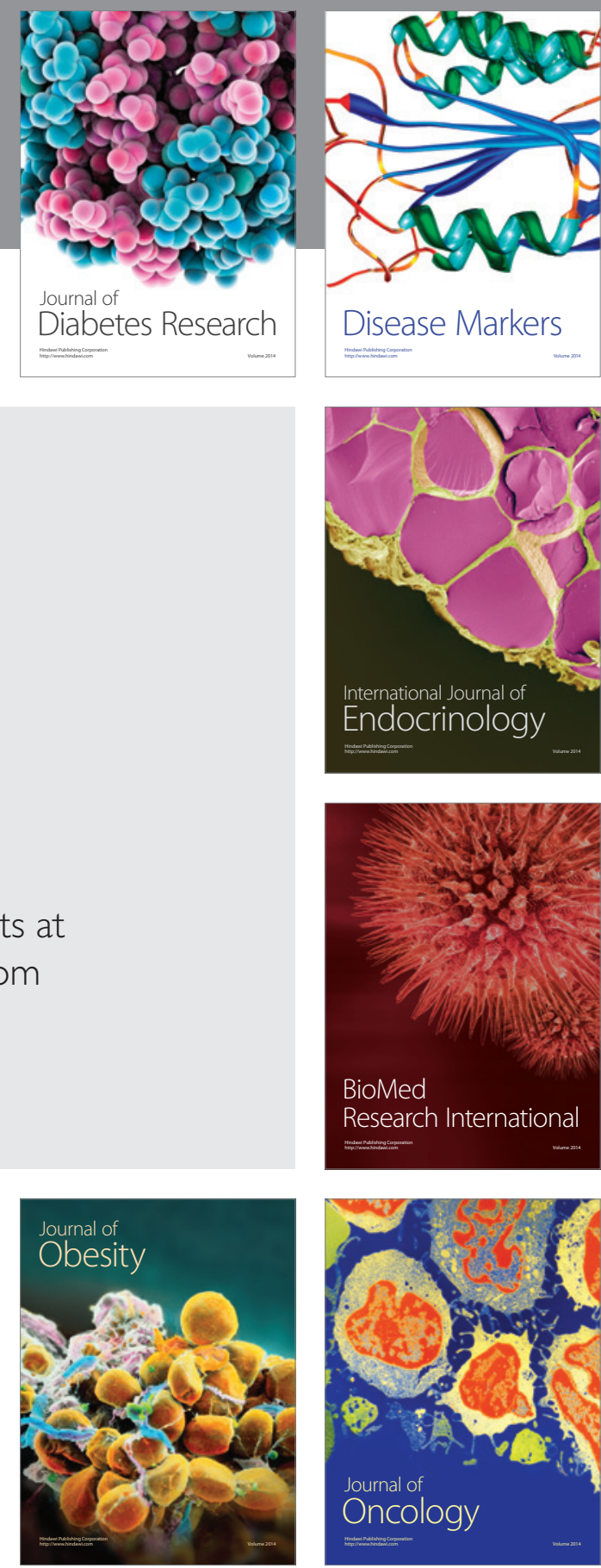

Disease Markers
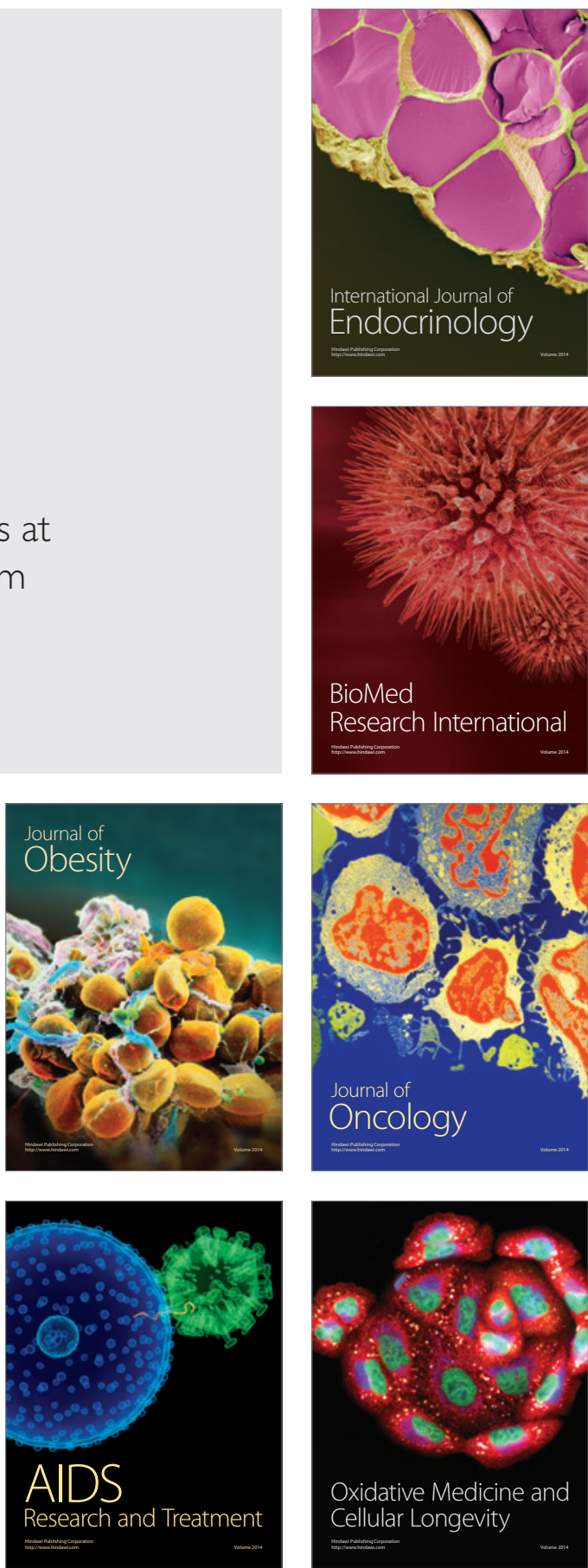